\title{
EMULSIÓNES TIPO PICKERING A BASE DE ALMIDONES MODIFICADOS COMO AGENTES EMULSIFICANTES. ARTÍCULO DE REVISIÓN
}

\author{
Pickering type emulsion based on StARCh LiKe EMUlsifying AGeNT. \\ REVIEW
}

Ana María Páez Álvarez *

Claudio Raúl Bernal Bustos**

Lina Hernández ${ }^{* * *}$

Lina Muñoz

Recibido: 15 de diciembre de 2017

Aceptado: 18 de mayo de 2018

\section{Resumen}

Este artículo de revisión propone una alternativa natural respecto a los agentes emulsificantes convencionales utilizados en los productos cosméticos, comparando las propiedades fisicoquímicas de los diferentes almidones utilizados en emulsiones tipo Pickering. Como resultado se elaboraron tres matrices conceptuales que generan información concluyente sobre las propiedades que debe tener un almidón para ser escogido como emulsificante, junto con las modificaciones químicas que le proporcionan un carácter anfifílico a dicha molécula, para finalmente encontrar las condiciones de elaboración de una emulsión Pickering.

Palabras clave: almidón, emulsión Pickering, modificación, quinua.

\begin{abstract}
This review proposes one natural alternative to replace the conventional emulsifying agents used in cosmetic products, comparing the physicochemical properties of the different starches used in Pickering emulsions. This article elaborated three conceptual matrices that provide information of the properties that a starch must have to be chosen as an emulsifier, likewise the chemical modifications that provide an amphiphilic character to said molecule; finally it find the conditions of elaboration of a Pickering emulsion.
\end{abstract}

Keywords: starch, Pickering emulsion, modification, quinoa.

\footnotetext{
* Estudiante del Programa Ingeniería Química, coinvestigador Centro BIQA-Almidones Funcionalizados (AL), Fundación Universidad de América, Bogotá, Colombia. ana.paez@estudiantes.uamerica.edu.co

${ }^{\star *}$ Estudiante doctorado (ED) en Avances en Ciencias y Biotecnología Alimentaria. Docente investigador, director del Centro de Bioprospección e Ingeniería Química Aplicada al Estudio de Biomoléculas e Industria (BIQA), Fundación Universidad de América, Bogotá, Colombia. https://orcid.org/0000-0002-0750-0142.

claudio.bernal@investigadores.uamerica.edu.co

*** Estudiante del Programa Ingeniería Química, semillero Centro BIQA-AL, Fundación Universidad de América, Bogotá, Colombia. lina.hernandez3@estudiantes.uamerica.edu.co

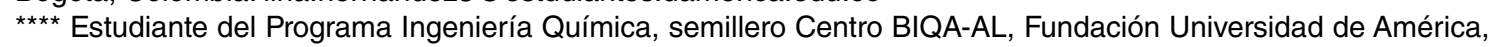
Bogotá, Colombia. lina.munoz@estudiantes.uamerica.edu.co
} 


\section{INTRODUCCIÓN}

Los productos cosméticos ${ }^{1}$ son sustancias aplicables a diferentes partes superficiales del cuerpo humano, cuyo fin exclusivo es modificar el aspecto, limpiar, perfumar, proteger o mantener la apariencia. Estos productos han sido usados desde la antigüedad hasta nuestros días. Si bien las mujeres han mostrado mayor interés por estas sustancias, los hombres están cada vez más interesados por su cuidado personal, por lo que son un mercado potencial de dichos productos (Tovar, Jaramillo y Toro, 2016).

Para garantizar que no existen efectos adversos en el uso de cosméticos, se realizan investigaciones con animales, los cuales son expuestos a dosis altas en periodos cortos, ocasionando que se aprueben cosméticos para el uso humano sin tener en cuenta los daños que estos podrían conllevar a largo plazo (Navarro, Núñez y Cebrián, 2012). Además, algunos de los compuestos utilizados en la industria cosmética son altamente peligrosos para la piel, incluso cancerígenos (Navarro et ál., 2012), como es el caso de los nitroalmizcles, policíclicos, ftalatos, nitrosaminas, compuestos orgánicos halogenados, parabenos, derivados de formaldehido, polietilenglicoles, entre otros.

La mayoría de los productos cosméticos son elaborados a partir de emulsiones, las cuales son una mezcla de dos fases inmiscibles, en la que una se encuentra dispersa en la otra en forma de pequeñas gotas. Estas fases deben ser estabilizadas para prevenir una coalescencia, es decir, que se vuelvan a unir las partículas en suspensión coloidal en forma de granos o gotas de mayor tamaño en una emulsión; por esto, la agitación es un factor relevante, debido a que entre mayor sea esta, menor será el tamaño de la partícula de la fase dispersa y mejorará su estabilidad (Moore y Wilkinson, 1990). Adicionalmente, para asegurar su estabilidad, se agrega a las dos fases inmiscibles un surfactante o emulsificante, que puede ser de origen natural, natural modificado o sintéticos (Gennaro, 2003), con el fin de disminuir la tensión superficial entre las fases y aumentar la repulsión electrostática entre las gotas (Rayner, Timgren, Sjöö y Dejmek, 2012). Los poliacrílicos son utilizados comúnmente para disminuir la tensión y aumentar la repulsión; no obstante, estas sustancias son tóxicas para la piel (Lehnmann, Volkert, Fischer, Schrader, y Nerenz, 2008), ya que vienen de un proceso de polimerización por adición, en el que quedan monómeros de ácido acrílico, provenientes del petróleo, que es altamente corrosivo y toxico. Por consiguiente, se ha encontrado una alta aplicabilidad de surfactantes poliméricos de origen natural, como los almidones, puesto que no tienen efectos secundarios para la piel; igualmente, proporcionan un buen aspecto a la emulsión, dado que son modificadores reológicos por naturaleza; sin embargo, esto depende del origen del almidón, debido a que la viscosidad se ve afectada por la relación amilosa-amilopectina (Goddard y Gruber, 1999).

Considerando lo expuesto, este artículo de revisión busca ampliar el enfoque científico sobre la elaboración de emulsiones de productos cosméticos, ya que son la fuente primaria de la mayoría de estos, en los que el componente principal es el agente tensoactivo (también llamado emulsificante). Para este el análisis se realiza un estudio de las características más relevantes que debe tener el emulsificante, especialmente cuando es de origen natural y puede sustituir a los emulsificantes sintéticos. Por otra parte, aquí se analizan las propiedades de cinco tipos de almidones utilizados en emulsiones Pickering, evidenciando que las características prevalentes del agente tensoactivo son el tamaño del grano y la relación amilosa-amilopectina que tenga el almidón, propiedades que influyen en la estabilidad de la emulsión cosmética.

\footnotetext{
${ }_{1}^{1}$ Para la definición de productos cosméticos, véase Comisión Europea (Directiva 76/768/CEE de 1976; Reglamento n. 1223 de 2009), Administración Nacional de Medicamentos, Alimentos y Tecnología Médica-ANMAT (Resolución 155 de 1998), sección 201 de la FDA (Cavers, 1938) y Real Decreto 944 de 2010 de España (citado en Sabater y Mourelle, 2013)
} 


\section{Almidón}

Los almidones por sí solos no son solubles en el agua y pueden variar el tamaño de granulo entre menos de $1 \mu \mathrm{m}$ hasta $100 \mu \mathrm{m}$, lo que proporciona características y utilidades diferentes (Lindeboom, Chang y Tyler, 2004). En la tabla 1 se evidencian las particularidades de cinco tipos de almidones naturales.

Tabla 1.Fuente de almidón según propiedades físicas

\begin{tabular}{lrrrrr}
\hline Fuente de almidón & Almidón \% & Amilosa \% & Amilopectina \% & $\begin{array}{r}\text { Temperatura } \\
\text { gelatinización }\end{array}$ & $\begin{array}{c}\text { Tamaño de } \\
\text { granulo } \\
\boldsymbol{\mu m}\end{array}$ \\
\hline Papa $^{\mathrm{a}}$ & $15-20$ & $21-25$ & $75-79$ & $59.3-67.8$ & 3.79 \\
Arroz $^{\mathrm{b}}$ & 80 & 17 & 83 & $68-78$ & $3-8$ \\
Yuca $^{\mathrm{c}}$ & $24-30$ & $25-30$ & $70-75$ & $60-67$ & 16 \\
Maiz $^{\mathrm{d}}$ & 88 & $20-30$ & $70-80$ & $62-65.3$ & 12 \\
Quinua $^{\mathrm{e}}$ & $52.5-69.2$ & 12.2 & 87.8 & 50.5 y 61.7 & $1-2$ \\
\hline
\end{tabular}

a. BeMiller y Whistler (2009), Nian, Bao, Bo y Han (2017), Pardo et ál. (2013), Wang (2017).

b. Ratnayake y Jackson (2003).

c. Jaramillo (1998), Meneses et ál. (2007), Morales (2014), Ruiz (2005).

d. Abugoch (2009), Narváez, Figueroa, Taba, Castaño y Martínez (2007), Pineda, Coral, Arciniegas, Rorales-Rivera y Rodríguez (2010), Wang et ál. (2011).

e. Abugoch (2009), Nair y Yamarik (2002), Marku et ál. (2012), Qian y Kuhn (1999).

Estas características ayudan a identificar y establecer un buen agente emulsificante natural que no afecte la piel ni al medio ambiente; por esta razón, se traen a consideración cinco tipos de almidones utilizados en la preparación de emulsiones Pickering.

\section{Almidón de papa}

La papa (Solanum tuberosum) tiene entre 15 y $20 \%$ de almidón en la totalidad del tubérculo (Pardo, Castañeda y Ortiz, 2013), lo que permite una adsorción de la fase oleosa y acuosa en la superficie del almidón, por lo que se puede observar una emulsión estable. Esto se logra a través de una modificación con octenil succinato anhídrido (OSA), el cual hace que tenga un carácter anfifílico y pueda adsorber fácilmente la fase acuosa (con lo que se puede obtener emulsiones W/O). Es fundamental evaluar el tamaño de partícula del almidón, ya que entre más pequeña sea la partícula, la emulsión será más compacta (según Wang [2017], el tamaño apropiado es 3.79 micrómetros). Cabe resaltar que el tamaño de partícula es inversamente proporcional a la estabilidad de la emulsión; de manera que con partículas pequeñas se obtiene una mayor eficiencia de empaquetamiento, así como una fase homogénea (Yusoff y Murray, 2010)

\section{Almidón de arroz}

$\mathrm{El}$ arroz (Oryza sativa) es una planta anual propia de terrenos muy húmedos, cuyo fruto es un grano oval rico en almidón, que es un alimento básico para más de la mitad de la población mundial (Food and Agriculture Organization of the United Nations, 1991). Este cereal viene en gránulos poliédricos con ángulos agudos y estrías concéntricas; tiene propiedades funcionales como espesante, gelificante y aglutinante, que permiten su uso en la industria cosmética; adicionalmente, el arroz suaviza la piel y es absorbente, lo que ayuda a formar una barrera protectora que evita el envejecimiento y la deshidratación de la piel. La desventaja de este producto es que se endurece al tener 
contacto con una atmósfera húmeda, por lo que bajo estas condiciones es un nutriente ideal para las bacterias, lo que causa la degeneración rápida del producto cosmético (López y Ozaeta, 2013).

La modificación con OSA se prepara mediante una reacción de esterificación estándar, en la que el anhídrido de ácido dicarboxílico cíclico y el almidón se suspenden en agua y se mezclan en condiciones alcalinas. El derivado obtenido de esta modificación tiene efectos emulsificantes $(\mathrm{He}$, Song, Ruan y Chen, 2006).

\section{Almidón de yuca}

La yuca (Manihot esculenta) tiene su origen en América Latina. Se cultiva de diferentes maneras y en diversos climas o condiciones de suelo. Normalmente, crece mejor en clima cálido y suelo argiloarenoso. Es conocida por su gran utilidad a nivel industrial para la obtención de almidón, bioetanol y otros productos de base biológica (por ejemplo, piensos, productos farmacéuticos, cosméticos y biopolímeros), debido a sus propiedades de retrogradación, menor temperatura de gelatinización (entre $60{ }^{\circ} \mathrm{C}-67{ }^{\circ} \mathrm{C}$ [Meneses, Corrales y Valencia, 2007]) y mayor capacidad de anclar las moléculas de agua junto con las de aceite (lo que forma micelas entre sí) respecto a otros almidones (Shubo et ál., 2017).

Se ha encontrado una posible aplicación del almidón de yuca en emulsiones cosméticas (ver Torrenegra, León, Matiz y Sastoque [2015]), por lo que, gracias a la liofilización con anhídrido dodecenil succínico, se logra generar un carácter hidrofóbico e hidrofílico que soporta las dos fases en una emulsión.

\section{Almidón de maíz}

El almidón de maíz (Zea mays) tiene aproximadamente un 20-30 \% de amilosa y 70-80 \% de amilopectina (Wang, Wang, Li, Zhou y Özkan, 2011). Este almidón ha sido utilizado en la industria alimenticia, médica y cosmética por su capacidad de desarrollar estructura de tipo gel cuando se enfría a temperatura ambiente. La gelificación y la cristalización tienen un aspecto irreversible dentro de la matriz de amilosa, mientras que la retrogradación a largo plazo de los geles de almidón es reversible, lo que puede atribuirse al contenido de amilopectina en el almidón (Miles, Morris, Orford y Ring, 1985).

Este almidón ha sido utilizado en emulsiones Pickering para el cuidado personal, en las que inicialmente se modifica con 1.2 epoxialcanos (C8-C12). Aquí se observa que, a medida que incrementa el número de carbonos en la cadena, el grado de sustitución disminuye, lo que hace desfavorable la estabilidad en la emulsión (Lehnmann et ál., 2008). También se observa que la transparencia de la emulsión es mayor a medida que aumenta la longitud de la cadena, por lo que se debe encontrar el equilibrio entre estas dos propiedades. Posteriormente, se realiza una reacción de eterificación con monocloroacetato, de la que resulta finalmente un almidón apto para ser utilizado como emulsificante con una estabilidad de 3 meses.

\section{Almidón de quinua}

El grano de quinua (Chenopodium quinoa) tiene entre el 52.5 \%-69.2 \% de almidón (Abugoch, 2009). Este almidón aumenta su poder anfifílico gracias a la modificación con OSA, por lo que es un buen prospecto para ser utilizado en emulsiones como emulsificante y estabilizante. Rayner et ál. (2012) concluyeron que el tamaño de granulo del almidón modificado es bueno como estabilizante de gotas de aceite. El tamaño de las gotas depende de la concentración de almidón que se le agregue; así, a mayor concentración de almidón, se observan menos cambios en el tiempo (su estabilidad está entre un día y una semana). Aunque este estudio se realizó con el fin de obtener 
una sustancia cremosa para la comida, se puede emplear en otras industrias, como la del papel, los cosméticos, las pinturas y los fármacos (Rayner et ál., 2012). Lo anterior lo confirma el estudio Timgren, Rayner, Dejmek, Marku y Sjöö (2012), el cual establece que el almidón de quinua es muy buen emulsificante y estabilizante gracias al tamaño pequeño de partícula. El almidón de quinua es un buen antioxidante por el alto contenido de fenoles que tiene, además de ser también antimicrobiano y proteger a la piel (Vilcacundo y Hernández-Ledesma, 2017).

En general, los gránulos de almidón se encuentran compuestos por amilosa y amilopectina; la primera sustancia les da su carácter amorfo, y la segunda, le proporciona su cristalinidad, haciendo que la relación entre ellas genere diferentes propiedades. Se ha observado que la temperatura de gelatinización del almidón se encuentra estrechamente relacionada con la amilopectina, ya que al aumentar la cristalinidad del grano, la temperatura de gelatinización debe ser más alta (Fredriksson, Silverio, Andersson, Eliasson y Åman, 1997); sin embargo, al igual que varios autores, Fredriksson et ál. (1997) no encuentran relación de la amilosa con la temperatura de gelatinización, ya que no mantiene un comportamiento uniforme con diferentes almidones; no obstante, se ha reportado que un contenido bajo de amilosa hace que la estructura cristalina se destruya fácilmente, permitiendo un alto poder de hinchamiento de granulo por su gran absorción de agua (Cheng, Mei y Kuo, 1996). Por lo anterior, es importante tener presente la relación amilosa-amilopectina, puesto que, para obtener una temperatura baja de gelatinización, los gránulos de almidón deben ser ricos en ramificaciones generadas por la amilopectina y tener una baja proporción en amilosa (Charles, Chang, Ko, Sriroth y Huang, 2005). Se ha observado que los gránulos de almidón de quinua tienen menor cantidad de amilosa y mayor concentración de amilopectina, por lo que tienen una temperatura de gelatinización baja, lo que es conveniente para no incurrir en costos energéticos adicionales; así, para asegurar una estabilidad futura, se recomienda calentar las fases (Duffus, Norton, Smith, Norton y Spyropoulos, 2016) para mantenerlas a la misma temperatura y evitar posibles choques térmicos que afecten la estabilidad de la emulsión. Además, la viscosidad depende de la amilosa; tal como lo afirman Charles et ál. (2005), a mayor cantidad de amilosa, menor será la viscosidad que se pueda lograr. Esto también es sustentado por el estudio de Jane et ál. (1999); según los autores, al incrementar el contenido de amilosa, decrece la viscosidad (Jane et ál., 1999), lo que muestra otra ventaja de trabajar con el almidón de quinua.

\section{Modificación del almidón}

Modificar el almidón permite disminuir la retrogradación y la tendencia de gelificación y mejora la claridad y textura en pastas y geles (Bemiller, 1997). El almidón modificado ha tenido una alta acogida por la diversidad de aplicaciones en industrias como la textil, la alimentaria y la cosmética, debido a que se han desarrollado varios métodos para modificar el almidón: químicos, físicos, enzimáticos y genéticos. Los métodos químicos son los más utilizados en la elaboración de emulsiones Pickering. Al realizar una modificación química se obtiene generalmente un producto de mayor resistencia a altas temperaturas, cizallamiento y $\mathrm{pH}$; también se consigue mayor viscosidad que con almidón sin modificar. Por ejemplo, cuando se hace reaccionar el almidón con ácidos orgánicos, su principal uso es la producción de materiales biodegradables. Asimismo, ayuda a la disminución de contaminantes por residuos sólidos (Peñaranda, Perilla y Algecira, 2008).

La modificación química se encuentra estrechamente relacionada con las reacciones de los grupos hidroxilos de la molécula de almidón, que generalmente se lleva a cabo por reacciones de eterificación, esterificación, entrecruzamiento, oxidaciones e injertos. Por ejemplo, el entrecruzamiento en almidones de tapioca con trimetafosfato de sodio ha demostrado un aumento en la viscosidad a causa de la presión osmótica causada por la presencia de sales (Varavinit, Paisanjit, Tukomane y Pukkahuta, 2007); por otro lado, si se realiza por medio de esterificación con ácido 
ferúlico, su viscosidad disminuye junto con su retrogradación, mientras que su capacidad de retener agua incrementa (Ou, Li y Yang, 2001).

La esterificación es una de las modificaciones más versátiles, pues incluye la introducción de ácidos orgánicos y derivados de estos. Los más utilizados para almidones son los donadores de acilo anhídrido o acilos clorados (Peñaranda et ál., 2008)

Debido a que son sustituciones que se realizan a la molécula de almidón, es necesario calcular el grado de sustitución que alcanza, el cual determina el número promedio de sustituciones en la unidad de anhidroglucosa (Peñaranda et ál., 2008). Para determinar este promedio exactamente, Plate, Diekmann, Steinhauser y Drusch (2011), entre otros autores, establecen dos métodos: acidificación y saponificación, para luego realizar la prueba de test de cloruros para determinar finalmente por titulación el grado de sustitución (Plate et ál., 2011).

\section{Emulsión Pickering}

Una emulsión está formada por dos líquidos inmiscibles entre sí que tienen una estabilidad homogénea gracias a los emulsificantes, que para el caso de este tipo de emulsiones son partículas sólidas. Dependiendo la distribución de las fases, cada emulsión tiene diferentes nombres: O/W significa que gotas de aceite se encuentran en agua (Song et ál., 2015), y W/O, que las gotas de agua se encuentra en la fase oleosa, que forma un ángulo mayor de $90^{\circ}$ al contacto con su emulsificante sólido (Aveyard, Binks y Clint, 2003; Marku, Wahlgren, Rayner, Sjöö y Timgren, 2012). El ángulo de contacto es importante, ya que influye en la energía de adhesión entre las moléculas de ambas fases y su surfactante (Marto et ál., 2015); además, muestra la relación superficial que permite determinar la afinidad del tensoactivo hacia alguna de las fases presentes.

Las emulsiones Pickering tienen grandes ventajas sobre las emulsiones convencionales; por ejemplo, alta estabilidad en contra de la coalescencia, lo que permite que coexistan altas concentraciones de la fase dispersa y en presencia de sales (Aveyard et ál., 2003); otra ventaja es que las partículas sólidas del emulsificante forman una barrera contra la transferencia de masa, efecto que los emulsificantes líquidos no hacen. Igualmente, estas emulsiones podrían utilizarse como vehículo para encapsular principios activos con entrega controlada (Frelichowska et ál., 2009).

Las emulsiones Pickering se separan si forman cremado o sedimentación. En el cremado, las partículas se ven desplazadas a la superficie de la solución debido a las diferencias de densidades, mientras que en la sedimentación pasa lo contrario, las partículas se desplazan al fondo del recipiente. Esto es influenciado por el diámetro de las partículas, la viscosidad del medio y las densidades de las fases. Sin embargo, los efectos mencionados no son tan graves como lo es la floculación y la coalescencia, ya que estos se pueden revertir con una agitación leve, mientras que en los últimos no es posible. La floculación es la agrupación de partículas (Fernández, 2006) causada por su potencial eléctrico; igualmente, la coalescencia es la fusión de estas, la cual depende de las características de la interface formada (Gennaro, 2003). Estas últimas partículas son las causantes de la ruptura de la emulsión en la mayoría de casos, ya que al formar grandes partículas se favorece la sedimentación y hace que se genere la separación de las fases.

\section{Metodología}

Este artículo de revisión propone una posible solución a los efectos secundarios que conllevan los productos cosméticos actuales del mercado, proporcionando información de un emulsificante natural a base de almidón que no afecte las características de la piel. Para llegar al resultado esperado, inicialmente se realizó el estado del arte sobre los almidones utilizados en emulsiones -preferiblemente 
cosméticas- para determinar las propiedades físicas, la modificación y las variables de proceso que incidan en la elaboración de las emulsiones y, asimismo, en la estabilidad de las mismas.

Se realizó una búsqueda exhaustiva por medio de las bases de datos a las que tiene acceso la Universidad de América, junto con la ayuda de los operadores booleanos (AND, XOR, NOT, OR) y tesauros para la construcción de frases claves de búsqueda, para una revisión adecuada de la información de los diferentes avances que se han reportado sobre el uso del almidón como posible agente emulsifcante. Posteriormente, se realizó un filtro de información y un análisis de los datos recolectados para plantear y estructurar las matrices comparativas de las propiedades físicas de los almidones que influyen en la emulsión, la modificación de los mismos y la elaboración de la emulsión tipo Pickering.

Finalmente, se realizó el análisis y las conclusiones de las matrices generadas que permitió determinar el almidón más favorable para la elaboración de emulsiones debido a sus propiedades físicas; seguidamente, se seleccionó el método de modificación que haya reportado el mayor grado de sustitución y las condiciones de proceso con las que se obtuvo una emulsión tipo Pickering estable en el tiempo.

\section{DesarRollo del tema}

Se estudian los emulsificantes naturales a base de almidón que se han investigado como sustituyentes de agentes tensoactivos sintéticos que causan efectos adversos a la piel (Lehnmann et ál., 2008). En la tabla 1 se reportan las principales propiedades físicas de los almidones: porcentaje de almidón, porcentaje de amilosa, porcentaje de amilopectina, temperatura de gelatinización y tamaño de granulo, las cuales se relacionan con la elaboración de emulsiones tipo Pickering en el tamaño de granulo y en la relación existente amilosa-amilopectina. Observando la matriz 1 (tabla 1), el tamaño de granulo oscila entre 1-16 $\mu \mathrm{m}$. Según la literatura, el gránulo del almidón de quinua es el de menor tamaño 1-2 $\mu \mathrm{m}$; sin embargo, los estudios previos del grupo de investigación Centro de Bioprospección e Ingeniería Química Aplicada al Estudio de Biomoléculas e Industria (BIQA) han encontrado tamaños de granulo entre $1.2 \mu \mathrm{m}$ y $1.8 \mu \mathrm{m}$ para variedades colombianas (Duarte, Bernal, y Ramírez, 2015), lo que favorece a la estabilidad de la emulsión, ya que evita la separación de la fase acuosa de la oleosa (Timgren et ál., 2012; Bello-Perez, Bello-Flores, Nuñez-Santiago, Coronel-Aguilera y Álvarez-Ramírez, 2015). Otra propiedad de gran importancia es la relación amilosa-amilopectina; según la literatura, la diferencia más significativa entre estas dos moléculas es el bajo contenido de amilosa y el alto contenido de amilopectina, lo que le proporciona a la emulsión mayor estabilidad, debido al poder de hinchamiento que adquiere el granulo respecto a su temperatura de gelatinización baja (Fredriksson et ál., 1997).

En el desarrollo de la matriz 2 (tabla 2) se encontró que es necesario realizar al almidón una modificación química que le proporciona a la molécula un carácter hidrofílico para que pueda ser utilizado como agente tensoactivo en una emulsión, especialmente con el reactivo octenil succinato anhídrido, el cual ha mostrado un alto grado de sustitución y un comportamiento favorable en emulsiones elaboradas previamente. 
Tabla 2. Fuente de almidones según tipo de modificación

\begin{tabular}{|c|c|c|c|c|c|}
\hline $\begin{array}{l}\text { Fuente de } \\
\text { almidón }\end{array}$ & Modificación & Reactivo para modificación & $\begin{array}{c}\text { \% W } \\
\text { reactivo / W } \\
\text { almidón }\end{array}$ & $\begin{array}{l}\text { Condiciones de } \\
\text { modificación }\end{array}$ & $\begin{array}{l}\text { Grado de } \\
\text { sustitución }\end{array}$ \\
\hline \multirow{3}{*}{ Maíz Ceroso a } & Eterificación & *1-2 epoxialcanos (C8-C12) y & - & ${ }^{*} 40^{\circ} \mathrm{C}$ y $3.5 \mathrm{~h}$ & 0.7 \\
\hline & & ácido monocloroacetico & & & \\
\hline & Esterificación ${ }^{b}$ & ${ }^{*}$ Octenil succinato anhídrido & - & ${ }^{*} \mathrm{pH} 7,62{ }^{\circ} \mathrm{C}$ y $5 \mathrm{~h}$ & * 0.022 \\
\hline Arroz $^{c}$ & Esterificación & Octenil succinato anhídrido & $5 \%$ & $\mathrm{pH} 8.4,35^{\circ} \mathrm{C}$ y $5 \mathrm{~h}$ & 0.0287 \\
\hline Yuca $^{d}$ & Lipofilizados & anhídrido dodecenil succínico & $10 \%$ & $\mathrm{pH} 8.5,25^{\circ} \mathrm{C}$ y $30 \mathrm{~min}$ & 0.35 \\
\hline $\mathrm{Papa}^{\mathrm{e}}$ & Esterificación & Octenil succinato anhídrido & $3 \%$ & $\mathrm{pH} 8.5,35^{\circ} \mathrm{C}$ y $2 \mathrm{~h}$ & - \\
\hline Quinua $^{\dagger}$ & Esterificación & Octenil succinato anhídrido & $4 \%$ & $\mathrm{pH} 7.8,1 \mathrm{~h}$ & 0.018 \\
\hline
\end{tabular}

a. Lehnmann et ál. (2008).

b. Huang et ál. (2010).

c. Song et ál. (2015).

d. Torrenegra et ál. (2015).

e. Wang (2017).

f. Timgren et ál. (2012).

Finalmente, en la matriz 3 (tabla 3) se reporta que (según el tipo de emulsión que se quiera obtener) tres variables afectan su estabilidad: porcentaje de emulsificante $(\% \mathrm{w} / \mathrm{w})$, fuente de almidón y condiciones de operación para preparar la emulsión.

Tabla 3. Fuente de almidón según la formulación de una emulsión Pickering

\begin{tabular}{|c|c|c|c|c|}
\hline Fuente De almidón & $\begin{array}{c}\text { Tipo De } \\
\text { emulsión }\end{array}$ & $\%$ W/W emulsificante & Estabilidad & Condiciones \\
\hline \multirow{2}{*}{ Maíz Ceroso ${ }^{\mathrm{a}}$} & $\mathrm{O} / \mathrm{W}$ & $3 \%$ & 3 meses & - \\
\hline & ${ }^{*} \mathrm{O} / \mathrm{W}{ }^{b}$ & * $7.5 \%$ & - & *16 000 rpm y 3 min \\
\hline Arroz $^{\mathrm{c}}$ & $\mathrm{O} / \mathrm{W}$ & $4 \%$ & 35 dias & 11000 rpm, 2 min y pH 6-7 \\
\hline Yuca $^{d}$ & $\mathrm{O} / \mathrm{W}$ & $2 \%$ & - & 1300 rpm y 3 min \\
\hline $\mathrm{Papa}^{\mathrm{e}}$ & $\mathrm{O} / \mathrm{W}$ & $2 \%$ & 2 meses & 150000 rpm y 3 min \\
\hline Quinua $^{f}$ & $\mathrm{O} / \mathrm{W}$ & $3.9 \%$ & 2 años & $11000 \mathrm{rpm}$ y $30 \mathrm{sec}$ \\
\hline
\end{tabular}
a. Lehnmann et ál. (2008).
b. Xu, Huang, Fu y Jane (2015).
c. Song et ál. (2015).
d. Torrenegra et ál. (2015).
e. Wang (2017).
f. Timgren et ál. (2012).

\section{Resultados}

Al analizar los almidones representativos en emulsiones Pickering, se evidencia que el almidón de quinua es el más adecuado para las emulsiones con alta estabilidad en el tiempo y para ser utilizado en emulsiones cosméticas, ya que cuenta con el menor tamaño de granulo, lo que permite que, cuando se forme la emulsión, sea más estable. Esta mayor estabilidad se debe a que la energía cinética de colisión es insuficiente para remover las partículas de la interface agua-aceite, por lo que no se evidencia la separación entre ellas. 
La relación amilosa-amilopectina es otra característica que muestra las ventajas del uso del almidón de quinua, ya que puede originar una temperatura de gelatinización baja, permitiendo un rápido poder de hinchamiento que facilita la interacción y el anclaje de las moléculas de aceite en agua. Además, esta relación evidencia un aumento en la viscosidad, que representa una rigidez entre las monocapas formadas en la emulsión, lo que genera estabilidad en el tiempo (Aveyard et ál., 2003), hecho que se reafirma con el comportamiento reológico típico que deben seguir las emulsiones Pickering (Matos et ál., 2018).

Finalmente, se encuentra en la literatura los rangos más adecuados para trabajar la elaboración de la emulsión: entre 2 a $7.5 \%$ peso a peso de emulsificante, agitación entre 11000 a 150000 y tiempo (aconsejable) de 3 minutos para garantizar la estabilidad de la emulsión, lo que le proporciona una textura cremosa y suave (Lindeboom, Chang y Tyler, 2004).

\section{Conclusiones}

Se concluye que las emulsiones Pickering a base de almidón son un buen sustituyente a los emulsificantes convencionales utilizados en productos cosméticos, ya que no presentan efectos adversos a la piel. Se verificó que las modificaciones químicas en las que se usa octenil succinato anhídrido como reactivo proporcionan una buena estabilidad a las emulsiones, pues permiten al almidón (normalmente insoluble en agua) ser hidrofílico e hidrofóbico al mismo tiempo y soportar la fase oleosa en su capa exterior, por lo que puede ser usado como emulsificante y estabilizante para las dos fases. Además, se logró identificar al almidón de quinua como potencial surfactante en emulsiones tipo Pickering, debido a dos de sus propiedades: tamaño de granulo y relación amilosaamilopectina, características que le confieren una alta estabilidad a la emulsión y un contenido bajo de amilosa, así como le proporcionan mayor viscosidad; sin embargo, es necesario encontrar las condiciones óptimas de operación para lograr el grado de sustitución requerido.

Por otro lado, para lograr una buena estabilidad entre la fase acuosa y oleosa, se encontraron que las variables de mayor influencia en la elaboración de la emulsión son la agitación y tiempo; no obstante, si es excesivo el tiempo de agitación, se destruirá nuevamente, por lo que no se lograría la emulsión deseada. Además, la concentración de almidón es de fundamental importancia, ya que ayuda a la estabilidad de la emulsión y hace posible la interacción de las dos fases, manteniéndolas relativamente unidas.

\section{RefERENCIAS}

Aveyard, R., Binks, B., y Clint, J. (2003). Emulsions stabilised solely by colloidal particles. Advances in Colloid and Interface Science, 100, 503-546.

Abugoch, L. (2009). Quinoa (Chenopodium quinoa Willd.): composition, chemistry, nutritional, and functional properties. Advances in food and nutrition research, 58, 1-31.

Bello-Pérez, L., Bello-Flores, C., Nuñez-Santiago, M., Coronel-Aguilera, C., y Alvarez-Ramirez, J. (2015). Effect of the degree of substitution of octenyl succinic anhydride-banana starch on emulsion stability. Carbohydrate Polymers, 132, 17-24.

Bemiller, J. (1997). Starch modification: Challenges and prospects. Starch-Stärke. 49(4), 127-131. http://dx.doi.org/10.1002/star.19970490402

BeMiller, J., y Whistler, R. (eds.). (2009). Starch chemistry and technology. EE. UU.: Elsevier.

Cavers, David, The Food, Drug, and Cosmetic Act of 1938: its legislative history and its substantive provisions. Law and Contemporary Problems, 6(1), 2-42. 
Charles, A., Chang, Y., Ko, W., Sriroth, K., y Huang, T. (2005). Influence of amylopectin structure and amylose content on the gelling properties of five cultivars of cassava starches. Journal of Agricultural and Food Chemistry, 53(7), 2717-2725.

Cheng, Y., Mei, T., y Kuo, T. (1996). Effect of amylose content on the rheological property of rice starch. Cereal Chemistry, 73(4), 415-420

Directiva 76/768/CEE del Consejo. Relativa a la aproximación de las legislaciones de los Estados miembros en materia de productos cosméticos. Comisión Europea 27 de julio de 1976.

Duarte, P., Bernal, C., y Ramirez, L. (2015). Obtención de carboximetil almidón de quinua, a escala laboratorio, a partir de almidón nativo de Chenopodium quinoa (Willd.) para su uso en la industria de hidrocarburos como aditivo en fluidos de perforación (tesis de pregrado). Facultad de Ingeniería Química, Fundación Universidad de América, Bogotá D. C., Colombia.

Duffus, L., Norton, J., Smith, P., Norton, I., y Spyropoulos, F. (2016). A comparative study on the capacity of a range of food-grade particles to form stable O/W and W/O pickering emulsions. Journal of Colloid and Interface Science, 473, 9-21.

Fernández, A. (2006). Preparación, caracterización y estabilidad de emulsiones y microemulsiones $\mathrm{O} / \mathrm{W}$ (tesis doctoral). Universidad de Granada, Granada, España.

Food and Agriculture Organization of the United Nations. (1991). El estado mundial de la agricultura y la alimentación. Roma: FAO.

Fredriksson, H., Silverio, J., Andersson, R., Eliasson, A., y Åman, P. (1997). The influence of amylose and amylopectin characteristics on gelatinization and retrogradation properties of different starches. Carbohydrate Polymers, 35(3-4), 119-134.

Frelichowska, J., Bolzinger, M., Valour, J., Mouaziz, H., Pelletier, J., y Chevalier, Y. (2009). Pickering w/o emulsions: Drug release and topical delivery. International Journal of Pharmaceutics, $368(1-2), 7-15$.

Gennaro, A. (2003). Remington: Farmacia (vol. 1). Buenos Aires: Editorial Médica Panamericana

Goddard, D., y Gruber, J. (Eds.). (1999). Principles of polymer science and technology in cosmetics and personal care. Nueva York: Marcel Dekker.

He, G., Song, X., Ruan, H., y Chen, F. (2006). Octenyl succinic anhydride modified early indica rice starches differing in amylose content. Journal of Agricultural and Food Chemistry, 54(7), 2775-2779.

Huang, Q. Fu, X., He, X., Luo, F., Yu, S., y Li, L. (20101). The effect of enzymatic pretreatments on subsequent octenyl succinic anhydride modifications of cornstarch. Food Hydrocolloids, 24(1), 60-65.

Jane, J., Chen, Y., Lee, L., McPherson, A., Wong, K., Radosavljevic, M., y Kasemsuwan, T. (1999). Effects of amylopectin branch chain length and amylose content on the gelatinization and pasting properties of starch1. Cereal Chemistry, 76(5), 629-637.

Jaramillo Diaz, H. (1998). Almidon de yuca (13ª ed.). Santiago de Cali: Universidad Autónoma de Occidente.

Lehmann, A., Volkert, B., Fischer, S., Schrader, A., y Nerenz, H. (2008). Starch based thickening agents for personal care and surfactant systems. Colloids and Surfaces A: Physicochemical and Engineering Aspects, 331(1-2), 150-154. 
Lindeboom, N., Chang, P., y Tyler, R. (2004). Analytical, biochemical and physicochemical aspects of starch granule size, with emphasis on small granule starches: a review. Starch-Stärke, 56(3-4), 89-99.

López, S., y Ozaeta, G. (2013). Extracción de almidón a partir de arroz de rechazo molido como viscosante en la elaboración de cinco cosméticos (trabajo de grado). Facultad de Ciencias Químicas y Farmacia, Universidad de San Carlos de Guatemala, Ciudad de Guatemala, Guatemala.

Marku, D., Wahlgren, M., Rayner, M., Sjöö, M., y Timgren, A. (2012). Characterization of starch pickering emulsions for potential applications in topical formulations. International Journal of Pharmaceutics, 428(1-2), 1-7.

Marto, J., Gouveia, L., Jorge, I., Duarte, A., Gonçalves, L., Silva, S., y Ribeiro, H. (2015). Starchbased pickering emulsions for topical drug delivery: a QbD approach. Colloids and Surfaces B: Biointerfaces, 135, 183-192

Matos, M., Laca, A., Rea, F., Iglesias, O., Rayner, M., y Gutiérrez, G. (2018). O/W emulsions stabilized by OSA-modified starch granules versus non-ionic surfactant: Stability, rheological behaviour and resveratrol encapsulation. Journal of Food Engineering, 222, 207-217.

Meneses, J., Corrales, C., y Valencia, M. (2007). Síntesis y caracterización de un polimero biodegradable a partir del almidón de yuca. Revista EIA, 8, 57-67.

Miles, M., Morris, V., Orford, P., y Ring, S. (1985). The roles of amylose and amylopectin in the gelation and retrogradation of starch. Carbohydrate research, 135(2), 271-281.

Moore, R., y Wilkinson, J. (1990). Cosmetología de Harry. Madrid: Ediciones Díaz de Santos. S. A.

Morales, E. (2014). Caracterización de películas, elaboradas a partir de harina de quinua (Chenopodium quinoa, Willd), almidón modificado de yuca (Manihot esculenta) y montmorillonita (tesis de pregrado). Facultad de Ingeniería Química y Agroindustria, Escuela Politécnica Nacional, Quito, Perú.

Nair, B., y Yamarik, T. (2002). Final report on the safety assessment of aluminum starch octenylsuccinate. International Journal of Toxicology, 21, 1-7.

Narváez, E., Figueroa, J., Taba, S., Castaño, E., y Martínez, R. (2007). Efecto del tamaño del granulo de almidón de maiz en sus propiedades térmicas y de pastificado. Revista Fitotecnia Mexicana, 30(3), 269-277.

Navarro, C., Núñez, M., y Cebrián, J. (2012). El libro de la cosmética natural. Barcelona: Ned ediciones.

Nian Zhou, Bao Zhang, Bo Chen, y Han- Qing Chen. (2017). Effects of oligosaccharides on pasting, thermal and rheological properties of sweet potato starch. Food Chemistry, 230, 516-523.

Ou, S., Li, A., y Yang, A. (2001). A study on synthesis of starch ferulate and its biological properties. Food Chemistry, 74(1), 91-95.

Pardo, O., Castañeda, J., y Ortiz, C. (2013). Caracterizacion estructural y térmica de almidones provenientes de diferentes variedades de papa. Acta Agronómica, 62(4), 289-295.

Peñaranda, O., Perilla, J., y Algecira, N. (2008). Revisión de la modificación química del almidón con ácidos orgánicos. Ingeniería e Investigación, 28(3), 47-52. 
Pineda-Gomez, P., Coral, D., Arciniegas, M., Rorales-Rivera, A., y Rodriguez, M. (2010). Papel del agua en la gelatinizacion del almidon de maiz: estudio por calorimetria diferencial de barrido. Ingenieria y Ciencia, 6(11) 129-141.

Plate, S., Diekmann, S., Steinhauser, U., y Drusch, S. (2011). Determination of the degree of substitution of hydrolysed octenylsuccinate-derivatised starch. Food Science and Technology, 46(2), 580- 580 .

Qian, J., y Kuhn, M. (1999). Characterization of Amaranthus cruentus and Chenopodium quinoa starch. Starch-Stärke, 51(4),116-120.

Ratnayake, W., y Jackson, D. (2003). Sources processing. En B. Caballero, Encyclopedia of Food Sciences and Nutrition (2a ed., 5567-5572). Nueva York: John Wiley \& Sons.

Rayner, M., Timgren, A., Sjöö, M., y Dejmek, P. (2012). Quinoa starch granules: A candidate for stabilising food-grade pickering emulsions. Journal of the Science of Food and Agriculture, 92(9), 1841-1847.

Reglamento (CE) n. ${ }^{\circ}$ 1223/2009. Sobre productos cosméticos. Diario Oficial de la Unión Europea L 342/59, 30 de noviembre de 2009.

Resolución 155/98. Productos cosméticos. Actualización de normas relacionadas con Productos Cosméticos para la Higiene Personal y Perfumes y las actividades inherentes a los mismos. Ministerio de Salud y Acción Social de Argentina, 13 de marzo de 1998.

Ruiz, G. (2005). Polímeros biodegradables a partir del almidón de yuca (tesis de maestría). Maestría en Ingeniería de Procesamiento de Polímeros, Universidad EAFIT, Medellín, Colombia.

Sabater, I., y Mourelle, L. (2013). Cosmetología para estética y belleza. Madrid: McGraw-Hill.

Shubo, L., Yanyan, C., Yuan, Z., Zhiting, L., Jidong, L., y Mouming, Z. (2017). The industrial applications of cassava: current status, opportunities and prospects. Journal of the Science of Food and Agriculture, 97(8), 2282-2290.

Song, X., Pei, Y., Qiao, M., Ma, F., Ren, H., y Zhao, Q. (2015). Preparation and characterizations of pickering emulsions stabilized by hydrophobic starch particles. Food Hydrocolloids, 45, 256-263

Timgren, A., Rayner, M., Dejmek, P., Marku, D., y Sjöö, M. (2012). Emulsion stabilizing capacity of intact starch granules modified by heat treatment or octenyl succinic anhydride. Food Science E Nutrition, 1(2), 157-171.

Torrenegra, M., León, G., Matiz, M., y Sastoque, J. (2015). Lipofilización del almidón de Dioscorea rotundata P. y su posible uso como agente emulsificante. Revista Cubana de Farmacia, 49(4), 605-617.

Tovar, M., Jaramillo, D., y Toro, G. (2016). Estudio de viabilidad para el desarrollo de una marca de cosméticos y productos de cuidado personal para hombres (trabajo de grado). Colegio de Estudios Superiores de Administración, CESA, Bogotá, Colombia.

Varavinit, S., Paisanjit, W., Tukomane, T., y Pukkahuta, C. (2007). Effects of osmotic pressure on the crosslinking reaction of tapioca starch. Starch-Stärke, 59(6), 290-296.

Vilcacundo, R., y Hernández-Ledesma, B. (2017). Nutritional and biological value of quinoa (Chenopodium quinoa Willd.). Current Opinion in Food Science, 14, 1-6. 
Wang, D. (2017). Food chemistry. New York: Nova Publishers.

Wang, L., Wang, B., Li, D., Zhou, Y., y Özkan, N. (2011). Shear-thickening properties of waxy maize starch dispersions. Journal of Food Engineering, 107(3-4), 415-423.

Xu, Y., Huang, Q. Fu, X., y Jane, J. (2015). Modification of starch octenylsuccinate by $\beta$-amylase hydrolysis in order to increase its emulsification properties. Food Hydrocolloids, 48, 55-61

Yusoff, A., y Murray, B. (2010). Modified starch granules as particle-stabilizers of oil-in-water emulsions. Food Hydrocolloids, 25(1), 42-55. 
